\title{
THE GERSCHGORIN DISCS UNDER UNITARY SIMILARITY
}

\author{
ANNA ZALEWSKA-MITURA and JAROSLAV ZEMÁNEK \\ Institute of Mathematics, Polish Academy of Sciences \\ P.O. Box 137, 00-950 Warszawa, Poland \\ E-mail: zemanek@impan.gov.pl \\ Fax: (48 22) 6293997
}

\begin{abstract}
The intersection of the Gerschgorin regions over the unitary similarity orbit of a given matrix is studied. It reduces to the spectrum in some cases: for instance, if the matrix satisfies a quadratic equation, and also for matrices having "large" singular values or diagonal entries. This leads to a number of open questions.
\end{abstract}

1. Motivation and the background. The unitary operators on finite-dimensional Hilbert spaces are distinguished among all linear operators by a number of remarkable properties. In particular, the unitary similarity orbit $\mathcal{U}(A)$ of a given operator $A$, that is, the set of operators of the form $U^{*} A U$, where $U$ is unitary, can shed light on the operator $A$ as well as on the structure of the group $\mathcal{U}$ of all unitary operators.

There are signs indicating the richness of the unitary group $\mathcal{U}$. For instance, the $S$ chur triangularization theorem [I. Schur 1909] guarantees the existence of a triangular matrix form (with respect to a given orthonormal basis) of a suitable member of $\mathcal{U}(A)$; the diagonal of this matrix gives the spectrum of $A$. The idea can be traced back to some earlier sources cited already by Schur, and even to Cauchy and Jacobi; for the history see e.g. [E. T. Browne 1958], [T. Hawkins 1975], [C. C. MacDuffee 1946], [L. Mirsky 1955], [H. W. Turnbull and A. C. Aitken 1932]. The standard simple proof has now a permanent place in textbooks (cf. [R. A. Horn and C. R. Johnson 1985], [V. V. Prasolov 1994]).

Also the singular values of $A$ can be seen within the unitary similarity orbit $\mathcal{U}(A)$ : there is a unitary $U$ such that the rows of (the matrix representation with respect to an orthonormal basis of) $U^{*} A U$ are orthogonal, and their Euclidean norms are the singular values of $A$ (see [E. T. Browne 1928]).

Every operator is a linear combination of four unitaries [A. G. Robertson 1974]. The whole algebra of operators is algebraically generated by two unitaries [C. Davis 1955],

1991 Mathematics Subject Classification: 15A18, 15A60.

The paper is in final form and no version of it will be published elsewhere. 
and is the linear span of $\mathcal{U}(A)$ in the case where $A$ is non-scalar with non-zero trace (see [B.-S. Tam 1986] and the references therein).

On the other hand, the closed connected group $\mathcal{U}$ seems to be distributed very economically on the unit sphere $\mathcal{S}$ of all operators having norm one. It is not difficult to see that the set $\mathcal{U}$ has no interior points with respect to $\mathcal{S}$, and that it does not cut the sphere $\mathcal{S}$ too much: the complement $\mathcal{S} \backslash \mathcal{U}$ remains connected. For each operator $A \in \mathcal{S}$ we have $\operatorname{dist}(A, \mathcal{U}) \leq 1$, with equality if and only if $A$ is non-invertible. No three distinct points of a unitary similarity orbit can lie on a real line [R. C. Thompson 1987]; this is also a consequence of the strict convexity of the Frobenius norm (and has an infinite-dimensional analogue [B. Aupetit, E. Makai, Jr. and J. Zemánek 1996]). An arbitrary neighbourhood of the identity contains two unitaries that generate a countable dense subsemigroup of $\mathcal{U}$ [H. Auerbach 1933].

The celebrated theorem of $S$. Gerschgorin [1931] says that the spectrum $\sigma(A)$ of an operator $A$ on an $n$-dimensional space is contained in the Gerschgorin region $\mathcal{G}(A)$, the union of the Gerschgorin discs

$$
\mathcal{G}_{i}(A):=\left\{\lambda \in \mathbb{C}:\left|a_{i i}-\lambda\right| \leq \sum_{j \neq i}\left|a_{i j}\right|\right\} \quad(i=1,2, \ldots, n),
$$

for each matrix representation $\left(a_{i j}\right)$ of $A$.

By considering the Jordan form of $A$, one can show that

$$
\sigma(A)=\bigcap_{T} \mathcal{G}\left(T^{-1} A T\right),
$$

where the intersection is taken over all invertible operators $T$ (cf. [R. A. Horn and C. R. Johnson 1985, Problem 6.1.2]). In other words, the spectrum of an operator $A$ is equal to the intersection of the Gerschgorin regions of $A$ considered with respect to all matrix representations of $A$. Thus, the Gerschgorin estimate is sharp in a natural way.

The following examples demonstrate the non-triviality of the estimate even for a single matrix.

EXAMPLE 1. If

$$
A=\left(\begin{array}{ll}
1 & 1 \\
1 & 1
\end{array}\right),
$$

then the spectrum $\sigma(A)=\{0,2\}$ lies on the boundary of the Gerschgorin region $\mathcal{G}(A)=$ $\mathcal{D}(1 ; 1)$.

EXAMPle 2. If

$$
A=\left(\begin{array}{cc}
-1 & 5 \\
1 & 1
\end{array}\right)
$$

then the spectrum $\sigma(A)=\{-\sqrt{6}, \sqrt{6}\}$ is contained in the first Gerschgorin disc $\mathcal{G}_{1}(A)$, but has no point in the smaller disc $\mathcal{G}_{2}(A) \subset \mathcal{G}_{1}(A)$.

The phenomenon pointed out in Example 2 cannot occur if the matrix $A=\left(a_{i j}\right)$ is normal. In that case, each of the Gerschgorin discs of $A$ must contain an eigenvalue of 
$A$; actually, even each of the smaller discs

$$
\left\{\lambda \in \mathbb{C}:\left|a_{i i}-\lambda\right| \leq\left(\sum_{j \neq i}\left|a_{i j}\right|^{2}\right)^{1 / 2}\right\} \subset \mathcal{G}_{i}(A) \quad(i=1,2, \ldots, n)
$$

must contain an eigenvalue of the normal matrix $A$ (see [V. Pták et J. Zemánek 1976, Théorème 2], [B. Aupetit 1991, Corollary 6.2.4], another proof is given in [A. Smoktunowicz 1996]). However, it is not true that the union of these smaller discs covers the spectrum, even for a self-adjoint matrix $A$, as the following example shows.

EXAMPLE 3. Let

$$
A=\left(\begin{array}{lll}
0 & 1 & 1 \\
1 & 0 & 1 \\
1 & 1 & 0
\end{array}\right)
$$

It is easy to verify that $(A+1)^{2}(A-2)=0$, hence $\sigma(A)=\{-1,2\}$. The eigenvalue 2 is on the boundary of the Gerschgorin region $\mathcal{G}(A)=\mathcal{D}(0 ; 2)$, but outside the smaller disc $\mathcal{D}(0 ; \sqrt{2})$. So the Gerschgorin theorem cannot be improved in this way, even for self-adjoint matrices. This leads to the following question.

QUESTION 1. Suppose that $\nu(\cdot)$ is a norm on $\mathbb{C}^{n-1}$ for which a Gerschgorin type theorem holds: For each $A \in M_{n}(\mathbb{C})$, the spectrum of $A$ is contained in the union of the discs

$$
\left\{\lambda \in \mathbb{C}:\left|a_{i i}-\lambda\right| \leq \nu\left(i \text {-th row of } A \text {, without } a_{i i}\right)\right\} \quad(i=1,2, \ldots, n) .
$$

Does it follow that $\nu(\cdot)$ majorizes the $l^{1}$-norm on $\mathbb{C}^{n-1}$ ?

Let us return to the sharpness with respect to similarity. The set of all invertible operators is too large compared to the compact set $\mathcal{U}$. In the present paper we intend to study the set

$$
\mathcal{Z}(A):=\bigcap_{U} \mathcal{G}\left(U^{*} A U\right)
$$

where the intersection is taken over all unitary operators $U$, and the Gerschgorin regions are considered with respect to an orthonormal basis. Clearly $\mathcal{Z}(A)=\sigma(A)$ for each normal operator $A$.

We shall show that $\mathcal{Z}(A)=\sigma(A)$ for each operator $A$ satisfying a quadratic equation (and thus, in particular, for each projection, see Sections 2 and 3). The result applies to operators having "large" singular values or diagonal entries (see Section 4). However, the set $\mathcal{Z}(A)$ may not reduce to the spectrum of $A$ in general, which leads to a number of interesting questions (Section 5).

It will be convenient to identify the operator $A$ with its matrix representation $\left(a_{i j}\right)$ with respect to an orthonormal basis $\left\{e_{1}, e_{2}, \ldots, e_{n}\right\}$. Thus, $a_{i j}=\left\langle A e_{j}, e_{i}\right\rangle$. In other words, the matrix

$$
\left(\begin{array}{cccc}
\left\langle A e_{1}, e_{1}\right\rangle & \left\langle A e_{2}, e_{1}\right\rangle & \ldots & \left\langle A e_{n}, e_{1}\right\rangle \\
\left\langle A e_{1}, e_{2}\right\rangle & \left\langle A e_{2}, e_{2}\right\rangle & \ldots & \left\langle A e_{n}, e_{2}\right\rangle \\
\ldots & & & \\
\left\langle A e_{1}, e_{n}\right\rangle & \left\langle A e_{2}, e_{n}\right\rangle & \ldots & \left\langle A e_{n}, e_{n}\right\rangle
\end{array}\right)
$$


represents the operator $U^{*} A U$ with respect to the standard basis $\{(1,0, \ldots, 0)$, $(0,1, \ldots, 0), \ldots,(0,0, \ldots, 1)\}$, where the columns of the unitary matrix $U$ are the $\mathbb{C}^{n_{-}}$ coordinates of the vectors $e_{1}, e_{2}, \ldots, e_{n}$.

It is interesting to note that every operator $A$ other than a multiple of the identity can have the above matrix representation with all entries different from zero for a suitable orthonormal basis [H. Radjavi and P. Rosenthal 1970, Theorem 2] and, moreover, $\max _{i, j}\left|\left\langle A e_{j}, e_{i}\right\rangle\right|$ is not constant over the family of all orthonormal bases [J. Dazord 1991, Théorème 3].

The numerical range $W(A)$ can be described, in our setting, as the set of complex numbers obtained at each (fixed) diagonal position, in all the above representations. On the other hand, the set of values occurring at each (fixed) off-diagonal position is clearly a closed disc centred at zero; the radius of this disc is equal to the distance

$$
\operatorname{dist}(A, \mathbb{C} I)=\inf _{\lambda \in \mathbb{C}}\|A-\lambda I\|=\max _{\|x\|=1}\left(\|A x\|^{2}-|\langle A x, x\rangle|\right)^{1 / 2},
$$

see [W. V. Parker 1948], [S. Prasanna 1981], [E. L. Stolov 1979]. For the Frobenius norm the corresponding distance can be expressed in terms of the trace [S. L. Lee 1996, Lemma 2.1], and this seems to be related to the estimate

$$
\left|\lambda-\frac{1}{n} \operatorname{tr} A\right| \leq \sqrt{\frac{n-1}{n}\left(\operatorname{tr} A^{*} A-\frac{1}{n}|\operatorname{tr} A|^{2}\right)} \quad(\lambda \in \sigma(A))
$$

obtained in [Y. Gu 1994].

Thus, in our problem, the centres of the Gerschgorin discs are restricted to the numerical range $W(A)$, and the radii cannot exceed $(n-1) \operatorname{dist}(A, \mathbb{C} I)$. Moreover, the Frobenius norm is constant on $\mathcal{U}(A)$, being equal to $\left(\operatorname{tr} A^{*} A\right)^{1 / 2}$. By [N.-K. Tsing 1983, Corollary 1], the farthest possible distance between two of the Gerschgorin centres, of a matrix in $\mathcal{U}(A)$, is diam $W(A)$. There are also bounds in terms of the singular values (see [R. A. Horn and C. R. Johnson 1991, pages 168, 188] and Section 4).

Concerning the size of the Gerschgorin region, there is the formula ([G. Dahlquist 1958], [S. M. Lozinskii 1958])

$$
\max \operatorname{Re} \mathcal{G}(A)=\lim _{t \rightarrow 0^{+}} t^{-1}(|I+t A|-1),
$$

where $|A|=\max _{i} \sum_{j}\left|a_{i j}\right|$. It resembles the formula [G. Lumer 1961]

$$
\max \operatorname{Re} W(A)=\lim _{t \rightarrow 0^{+}} t^{-1}(\|I+t A\|-1),
$$

where $\|\cdot\|$ is the operator norm.

It is also known [S. Gerschgorin 1931] that if $k$ of the Gerschgorin discs form a connected set, disjoint from the remaining $n-k$ discs, then this set contains precisely $k$ eigenvalues (counted with multiplicities) of the given matrix. Moreover, if an eigenvalue has $m$ linearly independent eigenvectors, then this eigenvalue lies in at least $m$ of the Gerschgorin discs [P. Stein 1952].

For us, the most intriguing situation happens when an eigenvalue appears on the boundary of the Gerschgorin region. If the given matrix is irreducible (that is, its permutation similarities are never block-triangular, see also [P. Lascaux et R. Théodor 1993] 
or [R. S. Varga 1962] for other equivalent definitions), then such an eigenvalue must be a common boundary point of all the Gerschgorin circles [O. Taussky 1948]. The latter property has the purely geometric consequence that the convex hull of the Gerschgorin centres (and thus, in particular, the arithmetic mean of the eigenvalues) is contained in the Gerschgorin region. It also implies restrictions on the coordinates of the corresponding eigenvector (cf. [R. A. Horn and C. R. Johnson 1985, Theorem 6.2.8]).

The Gerschgorin theorem had arisen from a number of sources, some of them are hardly available now. A historical account can be found e.g. in [E. Bodewig 1956], [E. T. Browne 1939], [A. S. Householder 1964], [E. H. Luchnis and M. A. McLoughlin 1996], [M. Marcus and H. Minc 1964], [W. V. Parker 1951], [M. Parodi 1959], and [O. Taussky 1949, 1962]. A conceptual proof embracing various localization results is discussed in [F. L. Bauer and C. T. Fike 1960] and [A. S. Householder 1964]. The analytic aspects are highlighted in [B. Aupetit 1991], [R. A. Brualdi and S. Mellendorf 1994], and [M. Newman 1980]. The connections with the numerical range are described in [K. E. Gustafson and D. K. M. Rao 1997] and [R. A. Horn and C. R. Johnson 1991]; see also [J. Dazord 1994] and [N. Nirschl and H. Schneider 1964]. For applications to the localization of the zeros of polynomials, see especially [H. E. Bell 1965]; it is interesting to compare this approach with the corresponding arguments based on the Rouché theorem in [N. Obreškov 1963] (see also [M. Marden 1966] and [M. Parodi 1959]). Needless to say, variants of the Gerschgorin theorem were also studied (see [R. A. Horn and C. R. Johnson 1985]).

Yet another kind of sharpness of the Gerschgorin estimate has been investigated in a series of papers initiated by [R. S. Varga 1965]. Also, there have been attempts to extend the Gerschgorin theorem to infinite-dimensional operators.

2. The two-dimensional case. To our surprise, the Gerschgorin theorem is sharp within the unitary similarity orbit of each $A \in M_{2}(\mathbb{C})$.

TheOrem 1. Let $A \in M_{2}(\mathbb{C})$. Then

$$
\bigcap_{U} \mathcal{G}\left(U^{*} A U\right)=\sigma(A)
$$

where the intersection is taken over all unitary matrices $U$ in $M_{2}(\mathbb{C})$.

Proof. By the Schur triangularization theorem, and after a suitable translation, rotation, and a further similarity by a diagonal unitary matrix, we may assume that

$$
A=\left(\begin{array}{cc}
\beta & 2 c \\
0 & -\beta
\end{array}\right),
$$

where $\beta \geq 0$ and $c>0$. We shall consider the three possible cases $\beta=0,0<\beta<c$, $\beta \geq c$, separately.

Case $\beta=0$. Consider the unitary matrix

$$
U=\frac{1}{\sqrt{2}}\left(\begin{array}{cc}
\omega & \omega \\
1 & -1
\end{array}\right)
$$


with $\omega \in \mathbb{C},|\omega|=1$. Then

$$
U^{*} A U=\left(\begin{array}{ll}
c \omega^{*} & -c \omega^{*} \\
c \omega^{*} & -c \omega^{*}
\end{array}\right)
$$

and the corresponding Gerschgorin discs touch each other at the origin, having centres at the points $\pm c \omega^{*}$. It is clear that the intersection of these regions, taken over all $\omega$ as above, yields $\{0\}$, the spectrum of $A$. (Notice that the Gerschgorin centres lie, in this case, on the boundary of the numerical range $W(A)=\mathcal{D}(0 ; c)$, an illustration of the result of Tsing mentioned in Section 1.)

Case $0<\beta<c$. The unitary matrices considered in the preceding case yield

$$
U^{*} A U=\left(\begin{array}{cc}
c \omega^{*} & \beta-c \omega^{*} \\
\beta+c \omega^{*} & -c \omega^{*}
\end{array}\right) .
$$

Now, the two Gerschgorin discs are again centred at $\pm c \omega^{*}$, and their radii are equal to the distance from the corresponding centre to the eigenvalue $\beta$. Thus, $\beta$ is a common boundary point of the two discs (cf. [O. Taussky 1948]). The intersection of all these regions, taken over $\omega \in \mathbb{C}$ with $|\omega|=1$, is the closed disc $\mathcal{D}(0 ; \beta)$ centred at 0 , with radius $\beta$. Indeed, let $|p|>\beta$. Let $S_{1} S_{2}$ be the diameter of the disc $\mathcal{D}(0 ; c)$ perpendicular to the line connecting $p$ with $\beta$. Then the point $p$ is avoided by the region consisting of the Gerschgorin discs centred at $S_{1}$ and $S_{2}$, since $\beta$ is their common boundary point.

It remains to reduce the $\operatorname{disc} \mathcal{D}(0 ; \beta)$ to the two-point set $\{ \pm \beta\}$, by using further unitary similarities. To this end, consider the unitary matrices

$$
U=\left(\begin{array}{cc}
\cos \alpha & \sin \alpha \\
-\sin \alpha & \cos \alpha
\end{array}\right)
$$

with $\alpha \in\left[-\frac{\pi}{4}, 0\right]$. Writing $t:=\sin 2 \alpha \leq 0$, we get

$$
U^{*} A U=\left(\begin{array}{cc}
\beta \sqrt{1-t^{2}}-c t & \beta t+c \sqrt{1-t^{2}}+c \\
\beta t+c \sqrt{1-t^{2}}-c, & c t-\beta \sqrt{1-t^{2}}
\end{array}\right):=\left(\begin{array}{cc}
S_{1}(t) & r_{1}(t) \\
r_{2}(t) & S_{2}(t)
\end{array}\right),
$$

where $t \in[-1,0]$. Observe that

$$
S_{1}(t)>0, \quad r_{1}(t)>0, \quad \text { while } \quad S_{2}(t)<0, \quad r_{2}(t) \leq 0 .
$$

Thus, the inequality

$$
S_{2}(t)-r_{2}(t)<S_{1}(t)-r_{1}(t)
$$

would guarantee that the corresponding Gerschgorin discs are disjoint and, consequently, that the set $\mathcal{Z}(A)$ certainly avoids the points $z \in \mathbb{C}$ with $\operatorname{Re} z$ strictly between the two sides of (4). Inequality (4) is equivalent to

$$
\left(c^{2}+\beta^{2}\right) t^{2}+2 c^{2} t+c^{2}-\beta^{2}<0,
$$

and this holds precisely when

$$
t \in\left(-1, \quad \frac{\beta^{2}-c^{2}}{\beta^{2}+c^{2}}\right) .
$$

For $t=-1$, the Gerschgorin discs are centred at $\pm c$, and they touch each other at the eigenvalue $\beta$. For $t=\left(\beta^{2}-c^{2}\right) /\left(\beta^{2}+c^{2}\right)$, the Gerschgorin discs are again centred at $\pm c$, but touching each other at the eigenvalue $-\beta$. For $t$ from the open interval (5), the 
Gerschgorin pair is moving continuously, from the first situation just described to the second, consisting of two disjoint discs all the time. This shows that each point of the set $\mathcal{D}(0 ; \beta) \backslash\{ \pm \beta\}$ will be avoided by some pair, for a suitable $t$ from $(5)$. Thus $\mathcal{Z}(A)=\sigma(A)$.

Case $\beta \geq c$. Consider the unitary matrix

$$
V=\frac{1}{\sqrt{\beta^{2}+c^{2}}}\left(\begin{array}{cc}
c & \beta \\
-\beta & c
\end{array}\right)
$$

whose first column is the eigenvector of $A$ corresponding to the eigenvalue $-\beta$, and the second column is chosen so that $V$ be unitary. Then

$$
V^{*} A V=\left(\begin{array}{cc}
-\beta & 2 c \\
0 & \beta
\end{array}\right)
$$

Consider the intersection of the Gerschgorin regions $\mathcal{G}(A)$ and $\mathcal{G}\left(V^{*} A V\right)$. If $\beta>2 c$, it already reduces to $\{ \pm \beta\}=\sigma(A)$. In the remaining case $c \leq \beta \leq 2 c$, this intersection consists of the points $\{ \pm \beta\}$ and a lens $\mathcal{L}$ (which reduces to $\{0\}$ if $\beta=2 c$, and is biggest if $\beta=c$ ). The family of matrices (2) satisfies (3) and (4), this time, for

$$
t \in\left(-\frac{2 \beta c}{\beta^{2}+c^{2}}, \quad 0\right) .
$$

Thus, each point of the set $\mathcal{L} \backslash\{ \pm \beta\}$ is omitted by the Gerschgorin region of (2), for a suitable $t$ from (6). The proof is complete.

It should be pointed out that the essential calculations behind the proof of Theorem 1 rely on the classical trigonometric formulas that are, by the way, consequences of the geometric fact

$$
\left(\begin{array}{cc}
\cos \beta & \sin \beta \\
-\sin \beta & \cos \beta
\end{array}\right)\left(\begin{array}{cc}
\cos \alpha & \sin \alpha \\
-\sin \alpha & \cos \alpha
\end{array}\right)=\left(\begin{array}{cc}
\cos (\alpha+\beta) & \sin (\alpha+\beta) \\
-\sin (\alpha+\beta) & \cos (\alpha+\beta)
\end{array}\right),
$$

a property of the unitary group! See, for instance, [V. Schranitzky 1996] or [K. Skurzyński 1996]. The unitary group of $M_{2}(\mathbb{C})$ is described in [R. L. Causey 1958].

3. Quadratic operators. Let $A$ be a linear operator on a (non-zero) finite-dimensional Hilbert space $X$. If the space decomposes into an orthogonal direct sum of $A$-invariant subspaces of dimension at most two, then we have $\mathcal{Z}(A)=\sigma(A)$, by applying Theorem 1 on the summands. We shall show that each operator satisfying a quadratic equation has this property.

Theorem 2. Suppose that $(A-\alpha)(A-\beta)=0$ on $X$, where $\alpha, \beta \in \mathbb{C}$. Then the operators $A$ and $A^{*}$ have a common invariant subspace of dimension 1 or 2 .

Proof. We note that also $\left(A^{*}-\alpha^{*}\right)\left(A^{*}-\beta^{*}\right)=0$, and the two factors commute. Since

$$
X=\operatorname{im}(A-\beta) \oplus(\operatorname{im}(A-\beta))^{\perp},
$$

where $\operatorname{im}(A-\beta) \subset \operatorname{ker}(A-\alpha)$ and $(\operatorname{im}(A-\beta))^{\perp}=\operatorname{ker}\left(A^{*}-\beta^{*}\right)$, it follows that

$$
X=\operatorname{ker}(A-\alpha)+\operatorname{ker}\left(A^{*}-\beta^{*}\right) .
$$


If there is a non-zero vector $x$ belonging to both the summands in (7), we have $A x=\alpha x$ and $A^{*} x=\beta^{*} x$, so that $x$ spans a one-dimensional common invariant subspace for $A$ and $A^{*}$.

It remains to consider the case where

$$
\operatorname{ker}(A-\alpha) \cap \operatorname{ker}\left(A^{*}-\beta^{*}\right)=0 .
$$

In view of $(7)$, one of these kernels must be non-zero, say $\operatorname{ker}\left(A^{*}-\beta^{*}\right) \neq 0$. Since $\operatorname{im}\left(A^{*}-\alpha^{*}\right) \subset \operatorname{ker}\left(A^{*}-\beta^{*}\right)$, it is clear that $\operatorname{ker}\left(A^{*}-\beta^{*}\right)$ is invariant under the self-adjoint operator $\left(A^{*}-\alpha^{*}\right)(A-\alpha)$. Consequently, $\operatorname{ker}\left(A^{*}-\beta^{*}\right)$ contains a non-zero eigenvector of $\left(A^{*}-\alpha^{*}\right)(A-\alpha)$ or, equivalently, $\operatorname{ker}\left(A^{*}-\beta^{*}\right)$ has a subspace $N$ of dimension 1 that is invariant under the operator $\left(A^{*}-\alpha^{*}\right)(A-\alpha)$. Let

$$
M=N+(A-\alpha) N \text {. }
$$

Then $M$ is invariant under $A-\beta$, because $(A-\beta)(A-\alpha) N=0$, and

$$
(A-\beta) N=((A-\alpha)+(\alpha-\beta)) N \subset(A-\alpha) N+N=M .
$$

Hence $A M=((A-\beta)+\beta) M \subset(A-\beta) M+\beta M \subset M+M=M$.

Moreover, $M$ is also invariant under $A^{*}-\beta^{*}$, because $\left(A^{*}-\beta^{*}\right) N=0$ (recall that $N$ is a subspace of $\left.\operatorname{ker}\left(A^{*}-\beta^{*}\right)\right)$, and

$$
\begin{aligned}
\left(A^{*}-\beta^{*}\right)(A-\alpha) N & =\left(\left(A^{*}-\alpha^{*}\right)+\left(\alpha^{*}-\beta^{*}\right)\right)(A-\alpha) N \\
& \subset\left(A^{*}-\alpha^{*}\right)(A-\alpha) N+\left(\alpha^{*}-\beta^{*}\right)(A-\alpha) N \\
& \subset N+(A-\alpha) N=M
\end{aligned}
$$

(recall that $N$ is invariant under $\left(A^{*}-\alpha^{*}\right)(A-\alpha)$ ). Thus, also $A^{*} M \subset M$.

Hence $M$ is invariant under both $A$ and $A^{*}$, and, of course, $1 \leq \operatorname{dim} M \leq 2$.

R e m a r k. Theorem 2 has been mentioned only recently in [H. Shapiro 1991, p. 159], it can also be derived from [T. J. Laffey 1981]. The simple proof given here is a slight modification of Solution 163 in [P. R. Halmos 1995]. Yet another simple proof can be found in [G. R. Allan and J. Zemánek, to appear], giving the same conclusion for every pair of quadratic operators (not just for a quadratic operator and its adjoint), even on a vector space as soon as the difference of the two operators has an eigenvector (which is automatically satisfied in the finite-dimensional case).

A common invariant subspace $M$, of an operator $A$ and its adjoint $A^{*}$, is called a reducing subspace of $A$. Equivalently, $M$ is a reducing subspace of $A$ if and only if $A M \subset M$ and $A M^{\perp} \subset M^{\perp}$. Consequently, the orthogonal complement of a reducing subspace is again a reducing subspace for the operator. Thus we get

Corollary 1. Let $A$ be a quadratic operator on a finite-dimensional Hilbert space. Then the space is an orthogonal direct sum of A-reducing subspaces of dimension at most two. In other words, the matrix of $A$ is unitarily similar to a block diagonal matrix with blocks of size at most two. In particular, $\mathcal{Z}(A)=\sigma(A)$.

It is well known that a rank one operator satisfies a quadratic equation (cf. [P. R. Halmos 1995, p. 239]). Thus we get

Corollary 2. If $\operatorname{rank} A=1$, then $\mathcal{Z}(A)=\sigma(A)$. 
We shall see, in Section 5, that the preceding conclusion may fail for operators of rank two. On the other hand, a projection, of any rank and not necessarily orthogonal, satisfies a quadratic equation, and thus, rather surprisingly, we obtain

Corollary 3. If $P^{2}=P$, then $\mathcal{Z}(P)=\sigma(P)$.

It seems worth noticing that the point here is the degree two of the minimal polynomial, not the angle between the range and kernel, nor their dimensions. By the way, quadratic operators are quite important in numerical analysis [N. Gastinel 1960].

T. J. Laffey has observed that if $A^{2}$ is normal, then $\mathcal{Z}(A)=\sigma(A)$, by applying Corollary 1 to the algebra generated by $A$ and $A^{*}$.

4. The Marcus-Sandy and Dazord inequalities. Let $\|A\|_{1}:=\operatorname{tr}\left(\left(A^{*} A\right)^{1 / 2}\right)$ denote the trace norm, that is, the sum of the singular values of an $n$ by $n$ matrix $A$. Let $w(A):=\max |W(A)|$ be the numerical radius, and let

$$
\|A\|_{\text {diag }}:=\max \left\{\left(\sum_{i=1}^{n}\left|b_{i i}\right|^{2}\right)^{1 / 2}: B=\left(b_{i j}\right) \in \mathcal{U}(A)\right\} \quad\left(\leq\|A\|_{1}\right) .
$$

The remarkable inequality found in [M. Marcus and M. Sandy 1985]

$$
\|A\|_{1} \leq n w(A)
$$

has been interestingly complemented in [J. Dazord 1995a]

$$
\|A\|_{1} \leq n^{1 / 2}\|A\|_{\text {diag }} \leq n w(A) .
$$

Moreover, it turns out [J. Dazord 1995a] that

$$
n^{1 / 2}\|A\|_{\text {diag }}=n w(A) \text { if and only if }\|A\|_{1}=n w(A) .
$$

This happens (cf. [M. Marcus and M. Sandy 1985], [J. Dazord 1995a]) if and only if $A / w(A)$ is unitarily similar to the direct sum of a diagonal unitary matrix and complex unit multiples of 2 by 2 matrices of the form

$$
\left(\begin{array}{cc}
1 & d \\
-d & -1
\end{array}\right)
$$

where $0<d \leq 1$. Consequently, Theorem 1 applies here:

Corollary 4. If $\|A\|_{1}=n w(A)$ or, equivalently, $\|A\|_{\operatorname{diag}}=n^{1 / 2} w(A)$, then $\mathcal{Z}(A)=$ $\sigma(A)$.

In this case, the matrix $B=\left(b_{i j}\right)$ realizing the maximum in (8) satisfies

$$
\left|b_{i i}\right|=n^{-1 / 2}\|A\|_{\operatorname{diag}}=n^{-1}\|A\|_{1}=w(A) \quad(i=1,2, \ldots, n),
$$

which is also proved in [J. Dazord 1995a] together with the following converse: If a matrix $A=\left(a_{i j}\right)$ satisfies $\left|a_{i i}\right|=w(A)$ for $i=1,2, \ldots, n$, then equality holds in (9). If only $\left|a_{i i}\right|=n^{-1 / 2}\|A\|_{\operatorname{diag}}(i=1,2, \ldots, n)$ is assumed, then equality holds in the first inequality in (10) [J. Dazord 1995b] .

These extremal properties imply geometric restrictions on the operators. For instance, if $\|A\|_{1}=n^{1 / 2}\|A\|_{\text {diag }}$ and $A \neq 0$, then $\operatorname{rank} A \geq n / 2$ [J. Dazord 1995b]. Thus, a projection $P$ of rank less than $n / 2$ cannot satisfy the assumptions of Corollary 4 , though we have $\mathcal{Z}(P)=\sigma(P)$ by Corollary 3 . 
Related results can also be found in [J. Dazord 1996], [R. Gabriel 1979], [L. László 1991, 1996, 1997].

\section{Further examples and questions}

EXAMPLE 4. Let

$$
A=\left(\begin{array}{lll}
0 & 1 & 0 \\
0 & 0 & 1 \\
0 & 0 & 0
\end{array}\right)
$$

in the standard basis of $\mathbb{C}^{3}$. Then $\mathcal{Z}(A)$ is a disc of positive radius, hence $\mathcal{Z}(A) \supsetneqq \sigma(A)$.

Proof. Let $\omega$ be a complex unit, and let $D=\operatorname{diag}\left\{1, \omega, \omega^{2}\right\}$. Then $D$ is unitary, and it is easy to check that $D^{*} A D=\omega A$ (cf. [T. Yoshino 1993, p. 132]). It follows that $\mathcal{Z}(A)=\omega \mathcal{Z}(A)$ for each complex unit $\omega$, hence $\mathcal{Z}(A)$ is a disc centred at zero. We shall show that the radius of this disc is strictly positive.

Let $r(U)$ denote the radius of the largest disc, centred at zero, that is contained in $\mathcal{G}\left(U^{*} A U\right)$. Since $r(U)$ depends continuously on $U$, which varies over the compact set $\mathcal{U}$, it is enough to show that $r(U)>0$ for each $U \in \mathcal{U}$. This is obvious if the Gerschgorin centres of $U^{*} A U$ are not collinear, since $\operatorname{tr} U^{*} A U=\operatorname{tr} A=0$ so that zero must be an interior point of $\mathcal{G}\left(U^{*} A U\right)$ in this case.

It remains to show that the three Gerschgorin discs of a $C \in \mathcal{U}(A)$ cannot touch each other at zero. Suppose it is the case, and let $a \geq b \geq c$ be the moduli of the Gerschgorin centres of $C$. Then each of these non-negative numbers is equal to the sum of the moduli of the two off-diagonal entries in the corresponding row, hence

$\left.\begin{array}{l}a^{2} \\ b^{2} \\ c^{2}\end{array}\right\} \geq \begin{aligned} & \text { the sum of the squares of the moduli of the off-diagonal entries in the cor- } \\ & \text { responding row of } C .\end{aligned}$

Moreover, the constancy of the trace and of the Frobenius norm on $\mathcal{U}(A)$ yields

$$
a=b+c,
$$

and

$$
a^{2}+b^{2}+c^{2} \geq 1
$$

We shall show that $c=0$, by finding successive upper estimates tending to zero. This will mean that the row corresponding to $c$ consists of zeros. Consequently, the principal 2 by 2 submatrix $B$ of $C$, formed by deleting the row and column corresponding to $c$, satisfies $\sigma(B)=0$, and hence $B^{2}=0$. The Gerschgorin theorem applied to $B$ implies that the two Gerschgorin discs of $B$ touch each other at zero (cf. [O. Taussky 1948, Theorem 2]), hence the column of $C$ corresponding to $c$ consists of zeros as well (otherwise zero would be in the interior of $\mathcal{G}(C)$ ). Thus, $C^{2}=0$, which is impossible because $A^{2} \neq 0$.

Since the diagonal entries of $C$ belong to the numerical range $W(A)=\{\lambda \in \mathbb{C}:|\lambda| \leq$ $\sqrt{2} / 2\}$ (cf. [T. Yoshino 1993, p. 134]), we have

$$
2 c \leq b+c=a \leq \sqrt{2} / 2,
$$


hence

$$
c^{2} \leq 1 / 8
$$

It follows from (11) that

$$
b^{2} \geq 1-a^{2}-c^{2} \geq 1-\frac{1}{2}-\frac{1}{8}=\frac{3}{8}
$$

hence $b \geq \sqrt{3 / 8}$.

Next, $c=a-b$ yields the better estimate

$$
c \leq \sqrt{1 / 2}-\sqrt{3 / 8}=(\sqrt{8}-\sqrt{6}) / 4 \quad(<\sqrt{1 / 8}) .
$$

This estimate can be improved further by iterating the preceding argument. Write

where

$$
c^{2} \leq \frac{(\sqrt{8}-\sqrt{6})^{2}}{16}=\frac{7-4 \sqrt{3}}{8}=\frac{1}{2}-\frac{x_{0}}{8}
$$

$$
x_{0}:=4 \sqrt{3}-3
$$

Let

$$
x_{n+1}:=4 \sqrt{x_{n}}-x_{n}=4-\left(2-\sqrt{x_{n}}\right)^{2} \quad(n=0,1,2, \ldots) .
$$

Then $0<x_{n}<x_{n+1}<4$ and, by induction,

$$
c^{2} \leq \frac{1}{2}-\frac{x_{n}}{8}
$$

for all $n$. The limit $x:=\lim x_{n}$ satisfies $x=4 \sqrt{x}-x$, hence $x=4$. Thus, (12) tends to $c^{2} \leq 0$. This completes the proof.

It is possible to show that $\mathcal{Z}(A) \varsubsetneqq W(A)$ in Example 4 .

Question 2. For which matrices $A$ do we have $\mathcal{Z}(A) \subset W(A)$ ? Does this inclusion possibly hold in general? Could [J. G. Stampfli and J. P. Williams 1968, Theorem 4] be used here?

QUESTION 3. Is it at least true that $\max |\mathcal{Z}(A)| \leq\|A\|$ ?

EXAMPLE 5. The matrix

$$
A=\left(\begin{array}{ccc}
0 & 1 & 1 \\
0 & 1 & 0 \\
0 & 0 & -1
\end{array}\right)
$$

has no pair of orthogonal eigenvectors, yet the eigenvalues \pm 1 are isolated points even of the single Gerschgorin regions $\mathcal{G}\left(V^{*} A V\right)$ or $\mathcal{G}\left(W^{*} A W\right)$, respectively, with the unitary matrices

$$
V=\frac{1}{\sqrt{2}}\left(\begin{array}{ccc}
\mathrm{i} & 1 & 0 \\
0 & 0 & \mathrm{i} \sqrt{2} \\
1 & \mathrm{i} & 0
\end{array}\right) \quad \text { and } \quad W=\frac{1}{\sqrt{2}}\left(\begin{array}{ccc}
1 & 1 & 0 \\
1 & -1 & 0 \\
0 & 0 & \sqrt{2}
\end{array}\right)
$$

Moreover, the unitary matrix

$$
U=\frac{1}{\sqrt{3}}\left(\begin{array}{ccc}
1 & -1 & 1 \\
-1 & (\sqrt{3}-1) / 2 & (\sqrt{3}+1) / 2 \\
1 & (\sqrt{3}+1) / 2 & (\sqrt{3}-1) / 2
\end{array}\right)
$$


yields

$$
U^{*} A U=\left(\begin{array}{ccc}
0 & 0 & 0 \\
-\frac{1}{\sqrt{3}} & -\frac{2}{\sqrt{3}} & -\frac{1}{\sqrt{3}} \\
-\frac{1}{\sqrt{3}} & \frac{1}{\sqrt{3}} & \frac{2}{\sqrt{3}}
\end{array}\right)
$$

so that the eigenvalue 0 is certainly a boundary point of the set $\mathcal{Z}(A)$; actually we do not know whether 0 is an isolated point of $\mathcal{Z}(A)$.

EXAmple 6. Replacing $1 / \sqrt{3}$ in the off-diagonal entries in the second and third rows of (13) by a positive number slightly less than $1 / \sqrt{3}$ (and retaining the signs as in (13)), we get a matrix $B$ whose all eigenvalues will be isolated points of the corresponding set $\mathcal{Z}(B)$, yet it is not clear whether $\mathcal{Z}(B)$ reduces to $\sigma(B)$.

The examples of this section lead to the following questions.

Question 4. Does $\mathcal{Z}(A)$ depend continuously on $A$ ? The answer is "yes" in the two-dimensional case, by Theorem 1 and the continuity of the spectrum.

QUestion 5. Is it true that each connected component of the set $\mathcal{Z}(A)$ must contain an eigenvalue of $A$ ?

Question 6. Characterize the operators A for which $\mathcal{Z}(A)=\sigma(A)$. More generally, characterize the eigenvalues of $A$ that are isolated (or boundary) points of the set $\mathcal{Z}(A)$.

QUESTION 7. Is the property $\mathcal{Z}(A)=\sigma(A)$ related to the proportion of the (suitable) norms of $A+A^{*}$ and $A-A^{*}$ ?

6. Conclusion. The Gerschgorin theorem is a rare instance of elegance and usefulness. We have tried to convey our belief that it could also contribute towards understanding the mystery of the unitary group. Our survey has been based on the dissertation [A. Zalewska-Mitura 1997].

7. Acknowledgements. We are grateful to a number of colleagues for interesting discussions over the years: the list should include M. Aleksiejczyk, G. R. Allan, T. Ando, B. Aupetit, L. Burlando, C. Davis, J. Dazord, L. Elsner, F. Hiai, C. R. Johnson, T. J. Laffey, L. László, C.-K. Li, Yu. Lyubich, E. Makai, Jr., A. Paszkiewicz, D. Petz, P. Rosenthal, A. Smoktunowicz, R. S. Varga, and L. Zsidó.

The paper has been brought into this volume, with great care, by Agnieszka Świạtkiewicz.

\section{References}

G. R. Allan and J. Zemánek [to appear], Invariant subspaces for pairs of projections, J. London Math. Soc.

H. Auerbach [1933], Sur le nombre de générateurs d'un groupe linéaire borné, C. R. Acad. Sci. Paris 197, 1385-1386.

B. Aupetit [1991], A Primer on Spectral Theory, Springer, New York. 
B. Aupetit, E. Makai, Jr. and J. Zemánek [1996], Strict convexity of the singular value sequences, Acta Sci. Math. (Szeged) 62, 517-521.

F. L. Bauer and C. T. Fike [1960], Norms and exclusion theorems, Numer. Math. 2, 137-141.

H. E. Bell [1965], Gershgorin's theorem and the zeros of polynomials, Amer. Math. Monthly $72,292-295$.

E. Bodewig [1956], Matrix Calculus, North-Holland, Amsterdam.

E. T. Browne [1928], The characteristic equation of a matrix, Bull. Amer. Math. Soc. 34, 363-368.

E. T. Browne [1939], Limits to the characteristic roots of a matrix, Amer. Math. Monthly 46, $252-265$.

E. T. Browne [1958], Introduction to the Theory of Determinants and Matrices, University of North Carolina Press, Chapel Hill, NC.

R. A. Brualdi and S. Mellendorf [1994], Regions in the complex plane containing the eigenvalues of a matrix, Amer. Math. Monthly 101, 975-985.

R. L. Causey [1958], Computing eigenvalues of non-Hermitian matrices by methods of Jacobi type, J. Soc. Indust. Appl. Math. 6, 172-181.

G. Dahlquist [1958], Stability and Error Bounds in the Numerical Integration of Ordinary Differential Equations, dissertation, Uppsala. Published in Kungl. Tekn. Högsk. Hand. Stockholm, No. 130, 1959.

C. Davis [1955], Generators of the ring of bounded operators, Proc. Amer. Math. Soc. 6, 970-972.

J. Dazord [1991], Sur une norme de matrices, C. R. Acad. Sci. Paris Sér. I Math. 312, 597-600.

J. Dazord [1994], On the C-numerical range of a matrix, Linear Algebra Appl. 212/213, 21-29.

J. Dazord [1995a], Une propriété extremale de la diagonale d'une matrice, lecture notes, Luminy.

J. Dazord [1995b], Matrices (1-d), lecture notes, Luminy.

J. Dazord [1996], Trace norm and spatial radius of a matrix, lecture notes, Chemnitz.

R. Gabriel [1979], Matrizen mit maximaler Diagonale bei unitärer Similarität, J. Reine Angew. Math. 307/308, 31-52.

N. Gastinel [1960], Utilisation de matrices vérifiant une équation de degré 2 pour la transmutation de matrices, C. R. Acad. Sci. Paris 250, 1960-1961.

S. Gerschgorin [1931], Über die Abgrenzung der Eigenwerte einer Matrix, Izv. Akad. Nauk SSSR 7, 749-754.

Y. Gu [1994], The distribution of eigenvalues of a matrix, Acta Math. Appl. Sinica 17, 501-511 (in Chinese).

K. E. Gustafson and D. K. M. Rao [1997], Numerical Range, Springer, New York.

P. R. Halmos [1995], Linear Algebra Problem Book, Mathematical Association of America, Washington, DC.

T. Hawkins [1975], Cauchy and the spectral theory of matrices, Historia Math. 2, 1-29.

R. A. Horn and C. R. Johnson [1985], Matrix Analysis, Cambridge University Press, Cambridge.

R. A. Horn and C. R. Johnson [1991], Topics in Matrix Analysis, Cambridge University Press, Cambridge.

A. S. Householder [1964], The Theory of Matrices in Numerical Analysis, Blaisdell, New York.

T. J. Laffey [1981], Algebras generated by two idempotents, Linear Algebra Appl. 37, 45-53.

P. Lascaux et R. Théodor [1993], Analyse Numérique Matricielle Appliquée à l'Art de l'Ingénieur 1, Masson, Paris.

L. László [1991], Upper bounds for matrix diagonals, Linear and Multilinear Algebra 30, 283301. 
L. László [1996], Upper bounds for the best normal approximation, lecture notes, Chemnitz.

L. László [1997], Upper bounds for the best normal approximation, preprint.

S. L. Lee [1996], Best available bounds for departure from normality, SIAM J. Matrix Anal. Appl. 17, 984-991.

S. M. Lozinskiu [1958], Error estimate for numerical integration of ordinary differential equations I, Izv. Vyssh. Uchebn. Zaved. Mat., no. 5 (6), 52-90; errata, 1959, no. 5 (12), 222 (in Russian).

E. H. Luchnis and M. A. McLoughlin [1996], In memoriam: Olga Taussky-Todd, Notices Amer. Math. Soc. 43, 838-847.

G. Lumer [1961], Semi-inner-product spaces, Trans. Amer. Math. Soc. 100, 29-43.

C. C. MacDuffee [1946], The Theory of Matrices, Chelsea, New York.

M. Marcus and H. Minc [1964], A Survey of Matrix Theory and Matrix Inequalities, Allyn and Bacon, Boston.

M. Marcus and M. Sandy [1985], Singular values and numerical radii, Linear and Multilinear Algebra 18, 337-353.

M. Marden [1966], Geometry of Polynomials, American Mathematical Society, Providence, RI.

L. Mirsky [1955], An Introduction to Linear Algebra, Clarendon Press, Oxford.

M. Newman [1980]. Geršgorin revisited, Linear Algebra Appl. 30, 247-249.

N. Nirschl and H. Schneider [1964], The Bauer fields of values of a matrix, Numer. Math. 6, 355-365.

N. Obreškov [1963], Zeros of Polynomials, Izdat. Bŭlgar. Akad. Nauk, Sofia (in Bulgarian).

W. V. Parker [1948], Sets of complex numbers associated with a matrix, Duke Math. J. 15, 711-715.

W. V. Parker [1951], Characteristic roots and field of values of a matrix, Bull. Amer. Math. Soc. 57, 103-108.

M. Parodi [1959], La Localisation des Valeurs Caractéristiques des Matrices et Ses Applications, Gauthier-Villars, Paris.

S. Prasanna [1981], The norm of a derivation and the Björck-Thomee-Istratescu theorem, Math. Japon. 26, 585-588.

V. V. Prasolov [1994], Problems and Theorems in Linear Algebra, American Mathematical Society, Providence, RI.

V. Pták et J. Zemánek [1976], Continuité lipschitzienne du spectre comme fonction d'un opérateur normal, Comment. Math. Univ. Carolin. 17, 507-512.

H. Radjavi and P. Rosenthal [1970], Matrices for operators and generators of $B(\mathcal{H})$, J. London Math. Soc. (2) 2, 557-560.

A. G. Robertson [1974], A note on the unit ball in $C^{*}$-algebras, Bull. London Math. Soc. 6, 333-335.

V. Scharnitzky [1996], Matrix Calculus, Müszaki Könyvkiadó, Budapest (in Hungarian).

I. Schur [1909], Über die charakteristischen Wurzeln einer linearen Substitution mit einer Anwendung auf die Theorie der Integralgleichungen, Math. Ann. 66, 488-510.

H. Shapiro [1991], A survey of canonical forms and invariants for unitary similarity, Linear Algebra Appl. 147, 101-167.

K. Skurzyński [1996], Elements of the theory of matrices, Gradient, no. 4, 216-234 (in Polish).

A. Smoktunowicz [1996], Remarks on inclusion theorems for normal matrices, lecture notes, Warszawa.

J. G. Stampfli and J. P. Williams [1968], Growth conditions and the numerical range in a Banach algebra, Tôhoku Math. J. 20, 417-424. 
P. Stein [1952], A note on bounds of multiple characteristic roots of a matrix, J. Research Nat. Bur. Standards 48, 59-60.

E. L. Stolov [1979], The Hausdorff set of a matrix, Izv. Vyssh. Uchebn. Zaved. Mat., no. 10, 98-100 (in Russian).

B.-S. Tam [1986], A simple proof of the Goldberg-Straus theorem on numerical radii, Glasgow Math. J. 28, 139-141.

O. Taussky [1948], Bounds for characteristic roots of matrices, Duke Math. J. 15, 1043-1044.

O. Taussky [1949], A recurring theorem on determinants, Amer. Math. Monthly 56, 672-676.

O. Taussky [1962], Eigenvalues of finite matrices: Some topics concerning bounds for eigenvalues of finite matrices, in: Survey of Numerical Analysis (ed. J. Todd), McGraw-Hill, New York, 279-297.

R. C. Thompson [1987], The matrix numerical range, Linear and Multilinear Algebra 21, 321-323.

N.-K. Tsing [1983], Diameter and minimal width of the numerical range, Linear and Multilinear Algebra 14, 179-185.

H. W. Turnbull and A. C. Aitken [1932], An Introduction to the Theory of Canonical Matrices, Blackie, London.

R. S. Varga [1962], Matrix Iterative Analysis, Prentice-Hall, Englewood Cliffs, NJ.

R. S. Varga [1965], Minimal Gerschgorin sets, Pacific J. Math. 15, 719-729.

T. Yoshino [1993], Introduction to Operator Theory, Longman, Harlow.

A. Zalewska-Mitura [1997], Localization of the Spectrum of Matrices by Means of Unitary Similarities, dissertation, Institute of Mathematics of the Polish Academy of Sciences, Warszawa (in Polish). 